NASA Technical Memorandum 109134

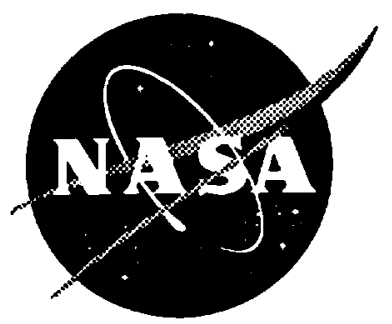

\title{
Real-Time RMS Active Damping Augmentation: Heavy and Very Light Payload Evaluations
}

Martha E. Demeo

VIGYAN, Inc., Hampton, Virginia

Michael G. Gilbert

NASA Langley Research Center, Hampton, Virginia

Janet A. Lepanto, and Karl W. Flueckiger

The Charles Stark Draper Laboratory, Inc., Cambridge, Massachusetts

Elizabeth M. Bains

NASA Johnson Space Center, Houston, Texas

$\begin{array}{lll}\infty & & \\ \infty & & \infty \\ m & n & N \\ m & 0 & n \\ 1 & n & m \\ j & U & 0 \\ 2 & 5 & 0\end{array}$

Mary C. Jensen

Lockheed Engineering and Sciences Company, Houston, Texas

June 1994

National Aeronautics and

Space Administration

Langley Research Center

Hampton, Virginia 23681-0001

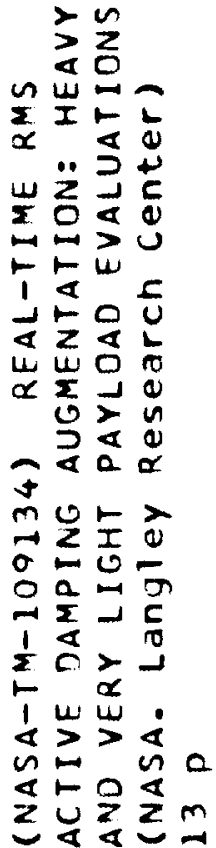




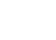

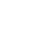




\title{
REAL-TIME RMS ACTIVE DAMPING AUGMENTATION: HEAVY AND VERY LIGHT PAYLOAD EVALUATIONS
}

\author{
Martha E. Demeo* \\ VíGYAN, Inc., Hampton, Virginia 23666 \\ Michael G. Gilbert ${ }^{\dagger}$ \\ NASA Langley Research Center, Hampton, Virginia 23681 \\ Janet A. Lepanto ${ }^{\ddagger}$ and Dr. Karl W. Flueckiger $\ddagger$ \\ The Charles Stark Draper Laboratory, Inc., Cambridge, Massachusetts 02139 \\ Dr. Elizabeth M. Bains $§$ \\ NASA Johnson Space Center, Houston, Texas 77058 \\ Mary C. Jensen \\ Lockheed Engineering and Sciences Company, Houston, Texas 77058
}

\begin{abstract}
Controls-Structures Integration Technology has been applied to the Space Shuttle Remote Manipulator System to improve on-orbit performance. The objective was to actively damp the undesired oscillatory motions of the Remote Manipulator System following routine payload maneuvering and Shuttle attitude control thruster firings. Simulation of Active Damping Augmentation was conducted in the real-time, man-in-the-loop Systems Engineering Simulator at NASA's Johnson Space Center. The simulator was used to obtain a qualitative definition of the Remote Manipulator System operational performance improvement from astronaut operators, and to obtain supporting quantitative performance data. Vibratory motions were computed using a threc-axis accelerometer simulation model located at the end of the lower boom of the Remote Manipulator System. The "sensed" motions were fed back to an Active Damping Augmentation control law, implemented in the Shuttle General Purpose Computer, which computed the joint servo mechanism commands to reduce the unwanted oscillations. Based on the request of astronaut operators who evaluated Active Damping Augmentation applied to an average (middle) weight payload, active damping of the Remote Manipulator System
\end{abstract}

* Research Engineer, Senior Member AlAA

$\dagger$ Assistant Branch Head, Spacecraft Dynamics Branch, Senior Member AIAA

$¥ \quad$ Senior Technical Staff, Control and Decision Systems Division

$\S$ Deputy Chief, Simulations Systems Branch, Associate Fellow AIAA

ๆ Test and Validation Engineer with attached heavy and very light payloads was demonstrated. Five astronaut operators examined the performance of two Active Damping Augmentation control laws which were implemented following single-joint and six-joint coordinated translational and rotational maneuvers. Active Damping Augmentation disturbance rejection of Shuttle thruster firings was also evaluated. Noticeable improvements in the damping response of the RMS with both the heavy, Hubble Space Telescope, payload and very light, astronaut in Manipulator Foot Restraint, payload were observed. The potential of Active Damping Augmentation to aid in maneuvering payloads close to structure or during other precision tasks was deemed significant.

\section{Introduction}

Active Damping Augmentation (ADA) is the application of Controls-Structures Integration (CSI) technology to benefit the on-orbit performance of the Space Shuttle Remote Manipulator System (RMS). The objective of ADA is to achieve measurable reductions in vibration decay times of the RMS which are significant and of direct visible benefit to RMS operators. Investigations into active damping of the RMS have been on-going for several years involving both industry and government participants [1-3]. The established approach to active damping augmentation is shown in Figure 1. Sensed vibratory motions are modeled using a simulated three-axis accelerometer package mounted near the wrist joints of the RMS. The sensed motions are fedback to the ADA control law, which is implemented in the Shuttle General Purpose Computer (GPC), to command selected joint servos reducing unwanted oscillations. The strategy for implementing ADA compensators in the RMS software has been to maintain the way the RMS "feels" to a trained operator, to localize the modifications to the greatest extent possible, and to adhere to the nominal health and safety monitoring of the RMS (including the payload dependent joint rate limits).

ADA has been applied to the RMS with the 3990 lb. Shuttle Pallet Satellite (SPAS), an average weight 


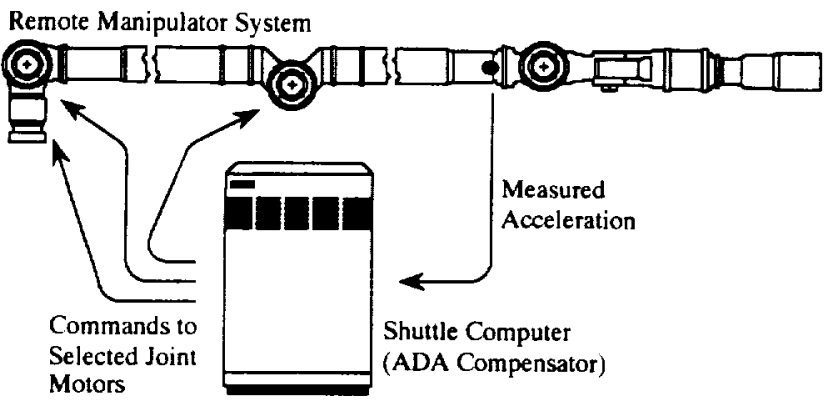

Figure 1: RMS ADA concept

attached payload, and the performance benefits of ADA evaluated in the real-time, human-in-the-loop, Systems Engineering Simulator (SES) at the NASA Johnson Space Center. In September 1992, astronaut operators assessed the performance of an ADA control law following single-joint and coordinated six-joint translational and rotational maneuvers, as well as ADA disturbance rejection of Shuttle thruster firings. Quantitative results were also derived from recorded simulator data. Significant reductions in the dynamic response of the $3990 \mathrm{lb}$. attached payload were observed. ADA benefits included reduced vibration decay time and peak response following RMS maneuvers and thruster firings, reduced RMS loads, improved payload positioning steady-state error, and transparency to the operator. Following this mid-weight payload evaluation, astronaut operators requested investigation of ADA application to heavier payloads where oscillations are of more concern. ${ }^{3}$

Based on this recommendation, as well as relevant mission requirements and engineering judgment, active damping of the RMS with attached heavy and very light payloads was investigated. ADA compensators, designed for the heavy and very light payloads, were implemented in the real-time SES and evaluation of ADA's performance improvement was obtained from trained RMS astronaut operators. This paper presents the results of this testing which demonstrates the range of payloads to which ADA may be applied and highlights respective benefits.

The paper is organized as follows. Section II provides a summary of the RMS system, the technical approach, study parameters used in the development of ADA control laws, and the objectives and goals for SES testing. Sections III and IV cover the development of the ADA control laws using identified system mathematical models and various control law design techniques. Section $V$ describes the SES RMS simulator, and Section VI presents the results of the testing.

\section{RMS ADA System}

\section{RMS System Description}

The Shuttle RMS is a 50-foot anthropomorphic arm which is used for the deployment, retrieval, and maneuvering of payloads. From its attachment point in the Shuttle cargo bay, the arm is comprised of two single degree-of-freedom (DOF) shoulder joints (yaw and pitch), a 21 -foot long upper boom, a single DOF elbow pitch joint, a 23-foot long lower boom, 3 single DOF wrist joints (pitch, yaw, roll) and a snare-type end effector capable of mating with a payload mounted grapple fixture. Encoders and tachometers reside at each of the six joint servos to measure joint position and joint rate, respectively. ${ }^{4}$

The arm is tele-robotically controlled from the aft cockpit of the Shuttle by translational and rotational hand controllers and control panel command inputs. Using various operational modes, joint angle readouts, remote cameras, and visual sightings, Shuttle astronauts maneuver the RMS to grapple and precisely position payloads for berthing, unberthing, and deployment. For various reasons, including stopping distance and easily excited RMS dynamics, the maneuvering of RMS-attached payloads is intentionally slow and deliberate. The dynamics of the RMS, which are low frequency and lightly damped, are largely due to structural flexibility of the RMS, the joint mechanisms and, to a lesser extent, the flexibility of Shuttle attachments. Significant nonlinearities in the joint mechanisms also exist.

\section{Technical Approach to ADA}

In this study, the approach to active damping augmentation of the RMS is to use feedback control laws to drive selected joints to suppress motion sensed by a threeaxis accelerometer mounted on the RMS. Initial work 1,2 described the use of the joint servo motors as actuators, the need for acceleration sensing, and possible control laws. As described in ref. [3], the strategy for implementing the ADA compensators was based on two ground rules. The first ground rule was that ADA not change the way the RMS "feels" to a trained operator. Thus, ADA is activated following the removal of RMS single joint and coordinated translational and rotational commanded motions. The second ground rule was that changes to the RMS software to accommodate ADA be localized to the greatest extent possible and adhere to the nominal health and safety monitoring of the RMS, including the payload dependent joint rate limits.

As in the mid-weight payload study, ${ }^{3}$ an important consideration in the implementation of ADA control laws in the Shuttle GPC was to minimize the inherent difficulties in multiple gain scheduling schemes necessary to accommodate maneuvers of the RMS over the workspace. To achieve this goal, several different robust control law design methods were used to establish candidate ADA control laws. The approach was to develop and test pointdesign control laws (control laws designed for a particular RMS configuration and payload) in the SES for damping performance and robustness to configuration changes. Several candidate control laws from this group were then further refined to achieve stable performance over a set of possible configurations and used for the astronaut evaluations reported in this paper. More detail on the design of the ADA controls laws is given in Sections III and IV.

\section{Study Parameters}

Fundamental parameters to this study involve Shuttle, payload, and RMS definitions. Shuttle and payload definitions consistent with the first HST Servicing Mission (SM) were selected. This mission included berthing the 


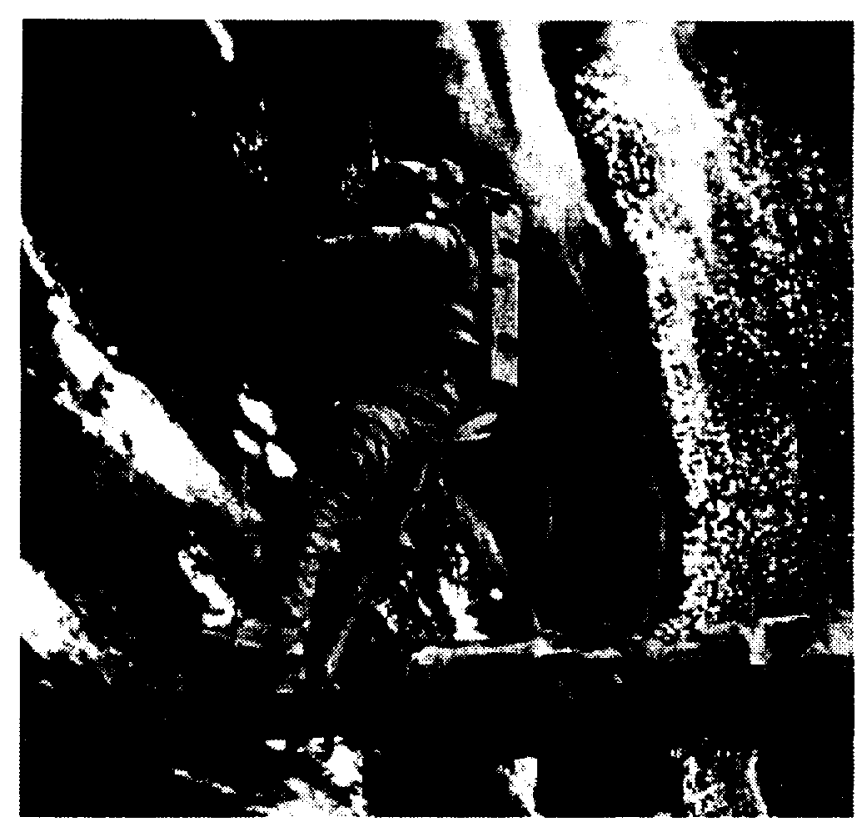

a) Astronaut in Manipulator Foot Restraint (MFR)

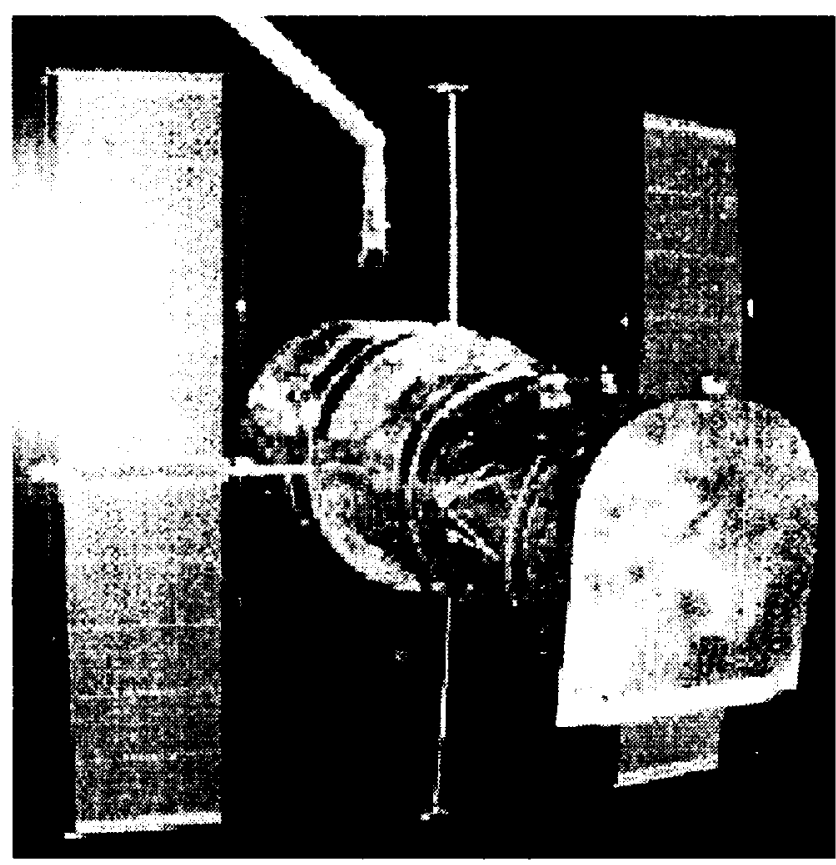

b) Hubble Space Telescope (HST)

Figure 2: MFR and HST Payloads

HST vertically in the aft section of the payload bay. In addition, numerous tasks were performed with an EVA astronaut mounted in the Manipulator Foot Restraint (MFR) on the end of the RMS. Photos of the HST and MFR candidate payloads are shown in Figure 2.

As with the mid-weight SPAS payload studies, a simulated three-axis accelerometer mounted at the end of the lower boom was used to sense the RMS vibration and provide feedback signals for ADA. The actuators selected for active damping include the shoulder yaw, shoulder pitch, and elbow pitch joint servos.
Point design arm configurations for the HST and MFR payloads were selected from the HST SM scenario. Figure 3 shows each of the payloads attached to the RMS in their respective target arm configurations.

\section{SYSTEM IDENTIFICATION}

In order to characterize the dynamics of the RMS with the HST and MFR payloads, several open-loop (i.e., without ADA) simulation runs were made in the SES. Plots of typical responses of each payload (including the midweight SPAS payload for reference) to a $10 \mathrm{sec}$ translation maneuver command are shown in Figure 4. The root-sumsquared (three-axis RSS) magnitude of the payload position is shown. The maneuvers were made at the maximum allowable rate for each of the payloads. These rate limits decrease with increasing payload mass as shown in Table 1. As a result, oscillations of the RMS with lighter payloads are more dramatic than with heavy payloads. In addition, the low frequency mode of the heavy HST payload has a corresponding period of $17.8 \mathrm{sec}$ as compared to only $3 \mathrm{sec}$ for the light weight MFR.

For the purposes of ADA control law design, linear multi-input/multi-output (MIMO) state space models were derived from simulated response data using the Observer/Kalman Filter Identification algorithm (OKID). ${ }^{7-9}$ This system identification methodology allows a state-space model and its corresponding observer to be identified directly from time response data.

Response data were generated in the SES using Preprogrammed Test Inputs (PTI's). Three PTI's were run for each of the three arm configurations which consisted of \pm full-scale rate command pulses to each of the shoulder yaw, shoulder pitch, and elbow pitch joint servos. The width of the command pulses was selected to correspond to the half-period of the fundamental mode of the arm with each of the attached payloads. This method of excitation was chosen over random noise or band-limited noise inputs to specifically excite the low frequency modes which were to be identified.

The inputs to the OKID algorithm were the joint rate commands to the shoulder yaw, shoulder pitch, and elbow pitch servos and the outputs were the simulated triaxial boom acceleration measurements. These acceleration measurements were filtered using third-order lowpass Butterworth filters in order to minimize aliasing, reduce effects of noise, and to provide a frequency weighted model. Filters with a break frequency of $0.06 \mathrm{~Hz}$ and 0.4 $\mathrm{Hz}$ were used for the HST and MFR payload models, respectively.

The identified discrete linear time-invariant plant models include the system dynamics and the filters in the form:

$$
\begin{gathered}
x(k+1)=A x(k)+B u(k) \\
y(k)=C x(k)+\operatorname{Du}(k)
\end{gathered}
$$

where $x(k) \in R^{n}, u(k) \in R^{m}$, and $y(k) \in R^{m}$. $A$ is the dynamics matrix; $B$ is the control distribution matrix, $C$ is the observation matrix; $\mathbf{D}$ is the control feedthrough matrix; 


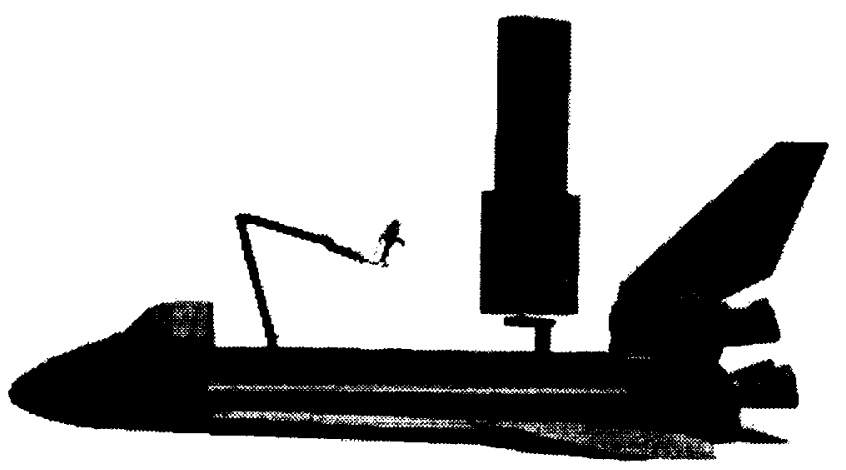

a) MFR scenario

(HST solar arrays not shown)

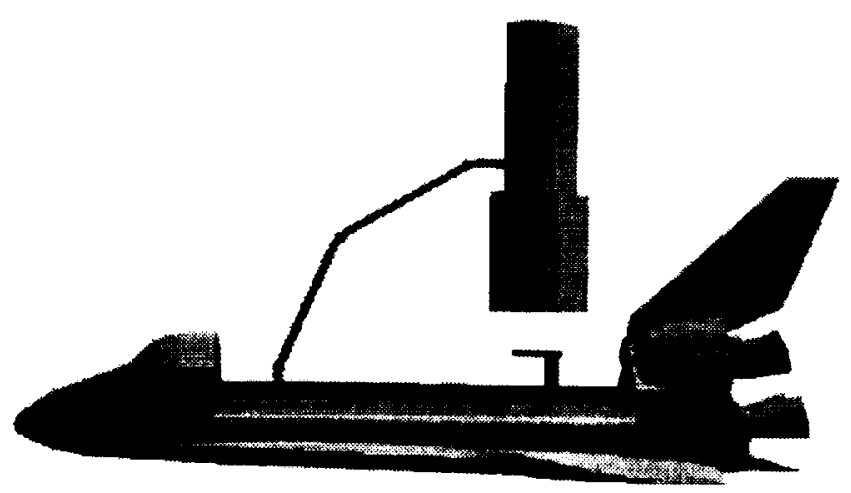

b) HST Scenario

(HST solar arrays not shown)

Figure 3: Scenario Descriptions

$\mathbf{x}(k)$ is the state vector; $\mathbf{u}(\mathrm{k})$ is the input state vector; and $\mathbf{y}(\mathbf{k})$ is the output vector. The identified model order, $n$, is specified by the user as part of the identification process. ${ }^{9}$

The corresponding identified observer obtained from the OKID algorithm is of the form:

$$
\begin{gathered}
\hat{\mathbf{x}}(k+1)=\mathbf{A} \hat{\mathbf{x}}(k)+\mathbf{B u}(k)-\mathbf{H}[\mathbf{y}(k)-\hat{\mathbf{y}}(k)] \\
\hat{\mathbf{y}}(\mathbf{k})=\mathbf{C} \hat{\mathbf{x}}(k)+\operatorname{Du}(k)
\end{gathered}
$$

where $\hat{x}(k)$ is the estimate of the state $x(k)$ and $H$ is the observer gain. Augmenting the plant model with this observer yields:

$$
\begin{gathered}
\hat{\mathbf{x}}(\mathbf{k}+1)=(\mathbf{A}+\mathbf{H C}) \hat{\mathbf{x}}(\mathrm{k})+[\mathbf{B}+\mathbf{H D}-\mathbf{H}]\left[\begin{array}{l}
\mathbf{u}(\mathrm{k}) \\
\mathbf{y}(\mathrm{k})
\end{array}\right] \\
\hat{\mathbf{y}}(\mathbf{k})=\mathbf{C} \hat{\mathbf{x}}(\mathrm{k})+\mathrm{Du}(\mathrm{k})
\end{gathered}
$$

This augmented system definition was used directly in the ADA compensator design. Twelfth-order linear models were identified for each of the point-design arm configurations. These models sufficiently captured the low frequency modes of the RMS/payload system.

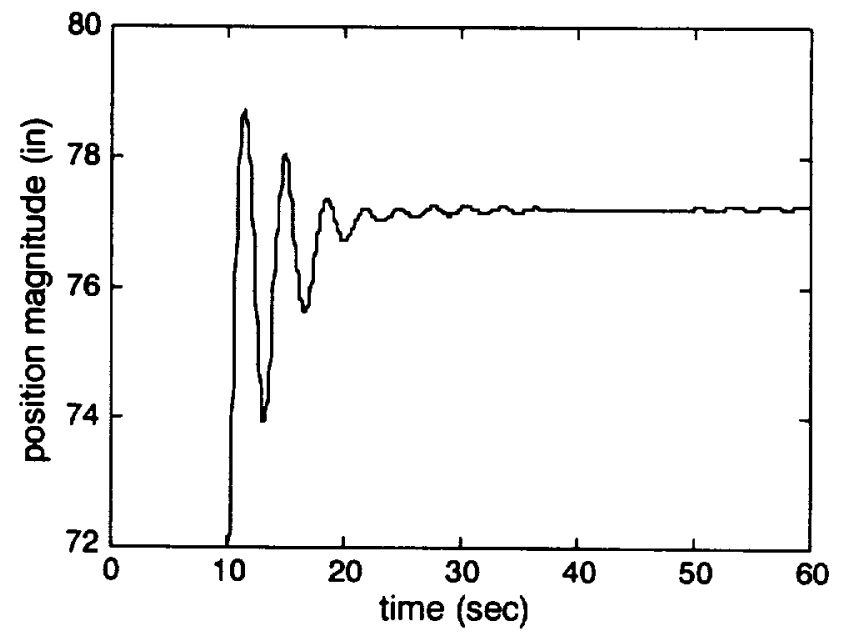

a) Astronaut in MFR (550 lbs.)

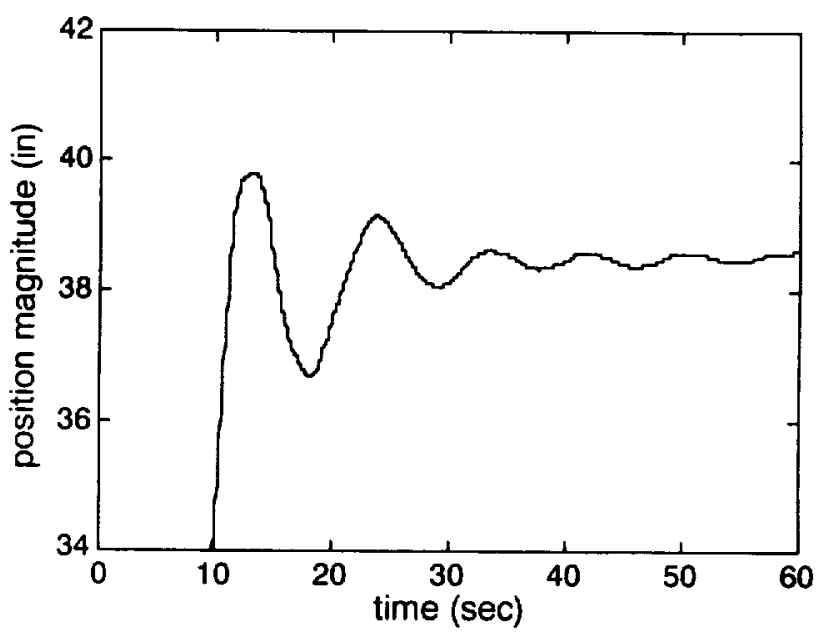

b) SPAS-02 (3,990 lbs.)

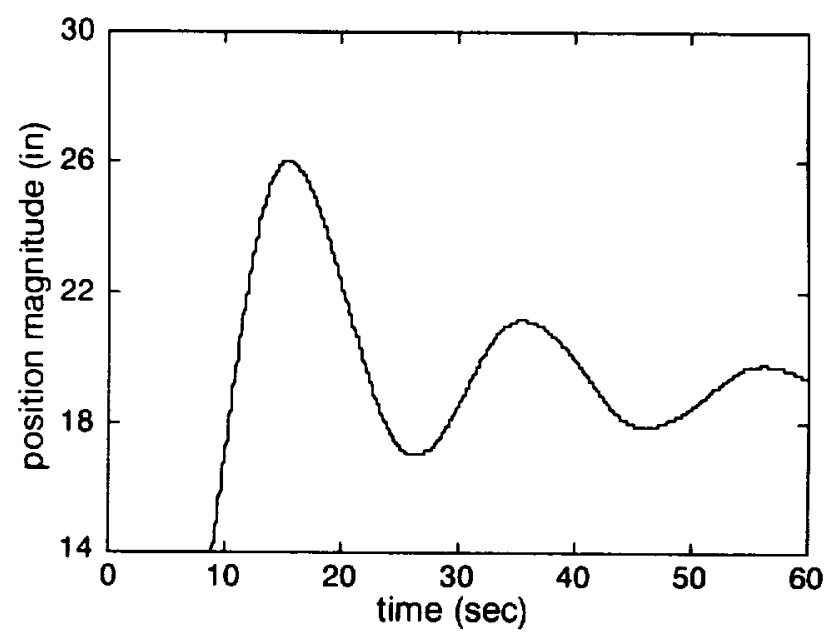

c) $\operatorname{HST}(23,900 \mathrm{lbs}$.

Figure 4: Typical Response to $10 \mathrm{sec} \mathrm{Y}$-axis Translation Maneuver 


\begin{tabular}{|c|c|c|c|c|}
\hline Payload & $\begin{array}{c}\text { Mass } \\
\text { (lbs) }\end{array}$ & $\begin{array}{c}\text { Frequency } \\
(\mathrm{Hz})\end{array}$ & $\begin{array}{c}\text { Maximum } \\
\text { Translational Rate } \\
(\mathrm{ft} / \mathrm{sec})\end{array}$ & $\begin{array}{c}\text { Maximum } \\
\text { Rotational } \\
\text { Rate (deg/sec) }\end{array}$ \\
\hline MFR & 550. & 0.33 & 0.77 & 2.79 \\
\hline SPAS-02 & $3,990$. & 0.16 & 0.36 & 1.47 \\
\hline HST & $23,900$. & 0.056 & 0.18 & 0.61 \\
\hline
\end{tabular}

Table 1: Respective Payload Rate Limits

\section{Control Law Design And Evaluation}

\section{Objectives and Approach}

The objectives of the -ADA-control law designs were to damp oscillations of the RMS system, i.e. to reduce the vibration decay time of the RMS following normal payload maneuvers and operations, and to be robust to changes in arm configurations. Two types of control laws were designed. The first were point-design compensators in which system nonlinearities were included as white noise parts of the OKID linear models, specifically in the observer noise properties. These nonlinearities included flexibility of structural members, joint servo and motor dynamics, gearbox compliance, and data transfer delays. The second type of control law was a single 'global' controller which addressed the kinematic nonlinearities of large joint angle maneuvers.

Two approaches were taken in the ADA compensator development. The first was an iterative LQRbased compensator and the second approach utilized robust multivariable techniques. The former was arbitrarily selected for use with the MFR model and the latter for the HST model. Each of the approaches started with control laws designed to operate at the respective point-design configurations and worked toward one that performed over the range of arm configurations in each of the respective scenarios.

Due to real-time implementation considerations, the maximum order of the compensator was constrained to be $\leq 24$. A discrete time representation of each compensator was implemented as shown in Figure 5.

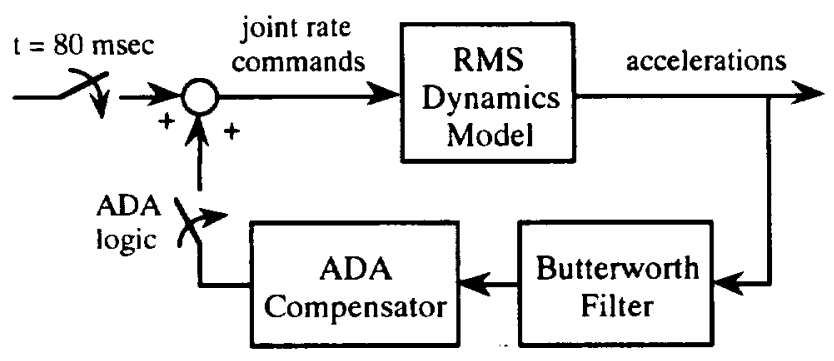

Figure 5: ADA Implementation

The ADA compensator was generically defined as:

$$
\begin{array}{r}
\mathbf{x}(k+1)=\mathbf{A}_{\mathbf{c}} \mathbf{x}(k)+\mathbf{B}_{\mathbf{c}} \mathbf{u}(k) \\
y(k)=\mathbf{C}_{\mathbf{c}} \mathbf{x}(k)+\mathbf{D}_{\mathbf{c}} \mathbf{u}(k)
\end{array}
$$

where Ac, Bc, Cc, and Dc are the compensator matrices. The inputs, $\mathbf{u}(\mathbf{k})$, are the three-axis filtered accelerations and the outputs, $y(k)$, are the joint rate commands to the shoulder and elbow servos.

The sections that follow provide descriptions of the LQR-based and the $H_{\infty} / \mu$-synthesis techniques used in design of control laws for the MFR and HST, respectively.

\section{MFR ADA Control Design}

A set of initial point design ADA compensators for the attached MFR were obtained using a frequency weighted LQR approach 10,11 and the identified (non-optimal) observer to provide state estimates. This is the same compensator design methodology which was employed in the mid-weight payload study ${ }^{3}$. This approach employed the discrete optimal control law,

$$
\mathbf{u}(\mathrm{k})=-\mathbf{G} \hat{\mathbf{x}}(\mathrm{k})
$$

where the mxn control gain matrix, $\mathbf{G}$, is defined as

$$
\mathbf{G}=\left(\mathbf{R}+\mathbf{B}^{\prime} \mathbf{K B}\right)^{-1} \mathbf{B}^{\prime} \mathbf{K A}
$$

and $\mathbf{K}=\mathbf{K}^{\prime}>0$ is an $\mathbf{n x n}$ solution matrix of the discrete algebraic Riccati equation. The regulator gain, $\mathbf{G}$, was be found to minimize the following quadratic cost function subject to the constraints imposed by the open-loop dynamics.

$$
\mathbf{J}=\sum_{\mathbf{k}=0}^{\infty}\left[\mathbf{x}^{\prime}(k) \mathbf{Q}^{*} \mathbf{x}(k)+\mathbf{u}^{\prime}(k) \mathbf{R}^{*} \mathbf{u}(k)\right]
$$

with $\mathbf{Q}^{*}=\mathbf{C}^{\prime} \mathbf{Q C}$ and $\mathbf{R}^{*}=\mathbf{D}^{\prime} \mathbf{Q D}+\mathbf{R}$. The output weighting matrix, $\mathbf{Q}^{*}$, and the control weighting matrix, $\mathbf{R}^{*}$, were assumed to be symmetric and positive semi-definite. Numerical values of $\mathbf{Q}$ and $\mathbf{R}$ were determined using an iterative design procedure on the linear model which avoided actuator saturation. That is, the required control was less than the payload dependent joint rate limits (e.g., for the shoulder joints and the MFR payload, this coarse mode limit was $1.35 \% \mathrm{sec}$ ).

The resulting MIMO ADA compensator equation was of the form:

$$
\begin{gathered}
\hat{\mathbf{x}}(\mathbf{k}+1)=(\mathrm{A}+\mathrm{HC}-\mathrm{BG}+\mathrm{HDG}) \hat{\mathbf{x}}(\mathrm{k})-\mathrm{Hy}(\mathrm{k}) \\
\mathbf{u}(\mathrm{k})=-\mathbf{G} \hat{\mathbf{x}}(\mathrm{k})
\end{gathered}
$$

where the compensator matrices from Eqn. 3.1 were defined as $A_{c}=(A+H C-B G+H D G), B_{c}=H, C_{c}=G$, and $D_{c}=0$. 


\section{HST ADA Control Design}

The compensator design approach used for the HST attached payload was based on robust multivariable synthesis techniques. The objective of this approach was to exploit the guarantee of performance specifications in the presence of modeling uncertainties while maintaining the same control architecture as the LQR/Observer design developed in the previous section.

Design of the robust controller began by converting the identified discrete time linear system model describing the HST hover position into the continuous domain, assuming the standard sample and zero order hold. The resulting open loop plant model is denoted by $\mathbf{G ( s )}=\mathbf{C}(\mathrm{sI}$ $\mathbf{A})^{-1} \mathbf{B}+\mathbf{D}$. An additive uncertainty, $\Delta \mathbf{G}(\mathrm{s})$, was also developed to characterize the large-angle manipulator dynamics, such that

$$
\mathbf{G}_{\text {true }}=\mathbf{G}(\mathbf{s})+\Delta \mathbf{G}(\mathbf{s})
$$

$\Delta \mathbf{G}(\mathrm{s})$ was assumed to be bounded by a fixed percentage $(25 \%)$ of the maximum singular value frequency response of the nominal model, $\mathbf{G}(\mathrm{s})$; at high frequencies $\Delta \mathbf{G}(\mathrm{s})$ was assumed bounded by $-60 \mathrm{~dB}$ times the infinity norm of $\mathrm{G}(\mathrm{s})$. This bound encompasses uncertainty due to potential mode shifts associated with changing RMS kinematics over the mission envelope as well as high frequency modeling uncertainties. The bound was approximated by a second order transfer function. The resulting system block diagram is given in Figure 6.

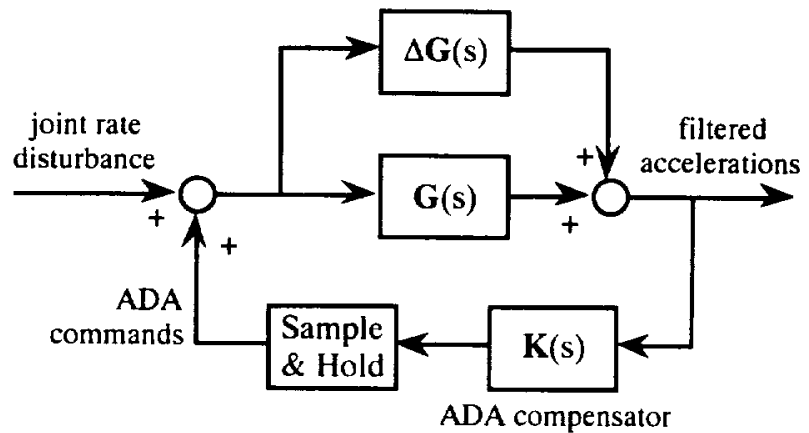

Figure 6: HST control design system model

By basic algebraic manipulations, an integrated model in standard form, as depicted in Figure 7, was assembled. The modeling error block, $\Delta$, was normalized to unit $H_{\infty}$ norm. The disturbance vector, d, reflects the external joint rate disturbance inputs. The error signal is given by $\mathbf{e}=\left[\mathbf{e}_{1}, \mathbf{e}_{2}\right]^{\mathrm{T}}$, where $\mathbf{e}_{1}$ is the (weighted) endeffector acceleration and $e_{2}$ consists of (weighted) joint rate commands. The systems weights were selected so that the disturbance is bounded by unity, $\|\mathbf{d}\|_{2}<1$, and performance specifications are met if $\|\mathrm{e}\|_{2}<1$. The fundamental design tradeoff was between the weighting associated with $e_{1}$ and $\mathbf{e}_{2}$. The two requirements, of ameliorating end-effector acceleration and maintaining reasonable (feasible) joint rates commands, by nature impose conflicting control system design objectives.

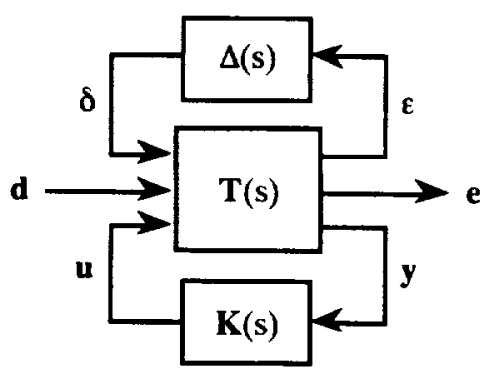

Figure 7: HST control design system in standard form

A $\mu$-analysis procedure ${ }^{12}$ was used to compute the desired control law. $\mu$-analysis incorporates information about the structure of the modeling uncertainty and performance requirements into the design procedure. If

$$
\|\mathbf{P}\|_{\mathrm{m}} \leq 1
$$

where $P$ is defined as the transfer function from $d$ to $e$ when the compensator is included in the feedback path from $y$ to $\mathbf{u}$ (see Figure 7), then for all unity norm bounded $\Delta$ (s) (with appropriate structure) both stability and performance specifications are achieved. 12

In general, it is not possible to compute $\mu$ precisely, and thus, a procedure was adopted in which $\mu$ was approximated by its upper bound satisfying:

$$
\mu(P)=\inf \bar{\sigma}\left(D_{P D}{ }^{-1}\right)
$$

where $\mathbf{D}$ is a real, frequency dependent, diagonal scaling matrix. ${ }^{12,13} \mu$-analysis computations provide not only basic analysis results (i.e., whether or not the system is robust), but also a set of frequency-dependent matrices, D, associated with each frequency at which $\mu$ is computed. The $\mu$-synthesis procedure ${ }^{14}$ uses these frequencydependent matrices to construct weighting matrices for an $\mathrm{H}_{\infty}$-optimal control problem that has a solution which may improve the subsequent stability and performance robustness of the resulting closed-loop control system.

Robust ADA controller development for the HST mission scenario required one $\mu$-synthesis iteration to generate a feasible design solution. The original $\mathrm{H}_{\infty}$ design was subjected to $\mu$-analysis, the frequency dependent $D$ weighting matrices were approximated by first order transfer functions, these transfer functions were appended to the design model and a second $\mathrm{H}_{\infty}$-optimal controller was generated. The resulting $\mathrm{H}_{\infty}$-optimal controller was reduced to 24 states using the balance and truncate technique of Moore. ${ }^{15}$. This reduction also has the beneficial numerical effect of balancing the compensator so that the resulting controller controllability and observability Gramians are diagonal and equal. Finally, the compensator was transferred back into the discrete domain.

\section{Evaluation}

Preliminary performance evaluations of the ADA point-design and global control laws were conducted using the identified linear models and additionally, two nonlinear simulations of the RMS. The two nonlinear simulations 
were the Draper RMS Simulator (DRS) and a modified version of the DRS called the SES/DRS. The DRS is a high fidelity non-real-time simulation of the RMS which has been validated against flight data. ${ }^{16}$ The simulation includes models of a rigid Shuttle, the seven links of the RMS and a rigid payload, as well as detailed models of the RMS joint servos and control software. The SES/DRS is a version of the DRS which mimics the dynamics of the realtime SES as well as computer signal time-delays.

Several evaluations were conducted with both the DRS and the SES/DRS to address maximum valid arm motion ranges, stability margins, variations between DRS and SES dynamics, gain scheduling, loads reductions, effects of RMS nonlinearities, and computer signal timedelays.

\section{Real-Time Simulation}

The Systems Engineering Simulator (SES) is a real-time, human and/or hardware-in-the-loop simulator located at the Johnson Space Center in Houston, Texas. It supports developmental testing and operations for Space Shuttle, Space Station Freedom, and advanced programs. Developmental testing typically includes evaluation of areas where human/machine interaction is a significant consideration. Operations support includes engineering, evolution, mission design and training, and real-time mission support when required.

Testing in the SES is typically performed in one or more of the available cockpit mockups. These mockups provide functional displays and controls for human-in-theloop interaction with a computer simulated dynamic environment. Out-the-window views and closed circuit television camera views, generated by electronic scene generators, give the operator visual cues that would be available in the environment being simulated. The on-orbit simulation capability of the SES using the Shuttle aft flight deck mockup, shown in Figure 8, was used for the RMS ADA study.

The on-orbit simulation for RMS ADA evaluation included the dynamics of the Shuttle with associated Flight Control System software, a high-fidelity dynamic RMS

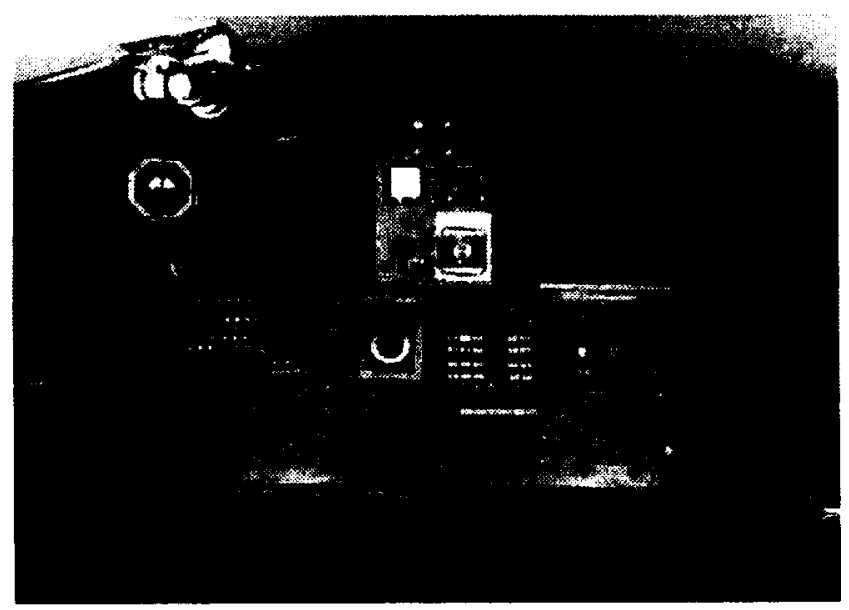

Figure 8. SES Shuttle aft flight deck model which has been validated against flight data ${ }^{17}$, and models of the HST and MFR payloads. The highest fidelity scene generator available was used to provide the maximum visual cues for damping evaluation. Models of an accelerometer on the RMS and the ADA control algorithm were added to SES as described in ref. [3].

Operator controlled ADA activation and deactivation from the cockpit was provided for engineering assessment. An existing switch in the aft cockpit mockup of the SES was adopted for this purpose. ${ }^{18}$

\section{Results}

Following preliminary evaluation of the ADA control laws using the non-real-time simulations, an engineering session was held in the SES to evaluate the realtime implementation of the candidate control laws and to make final gain adjustments. Noticeable improvements in the damping response of the RMS with both the heavy and very light payloads were observed in the scene generated window view and in the recorded simulation data.

Five astronaut operators and one JSC engineer were then invited into the SES to qualitatively evaluate the effectiveness and utility of ADA to on-orbit RMS operations. Following an informal briefing on the active damping augmentation methodology and an overview of the mid-weight payload evaluation results, the operators individually participated in real-time human-in-the-loop evaluation of ADA. Each of the evaluation sessions was broken into two task scenarios. The first scenario consisted of maneuvering the MFR payload from one worksite to the next, while the HST was berthed to a flight support system (FSS) in the aft end of the payload bay. The second scenario involved maneuvering the HST payload from the Extended Park configuration to a hover configuration just above the FSS. It was suggested that the operators maneuver the payloads from their initial configurations to the target configurations using manual and single joint mode commands and to observe the damping response with and without ADA. They were also encouraged to maneuver over the range of RMS configurations using arbitrary command inputs and Shuttle thruster inputs to excite the system with and without ADA active.

\section{Qualitative results}

In evaluating ADA, each of the astronauts maneuvered the RMS through a variety of configurations and command sequences. The astronauts' assessment of ADA for the MFR and HST payloads was generally favorable. As with the mid-weight payload evaluations, some operators were reluctant to deviate from established operating practices, which are related to on-orbit flight rules. Others were willing to exceed normal on-orbit operational practices for the purpose of evaluating ADA in the SES.

Plots of the responses to three survey questions which were posed to the astronauts are shown in Figure 9. Each operator was asked to select if they felt the topic was critical, significant, marginal, or insignificant. In some instances, astronauts gave an intermediate rating. As can be seen in Figure 9, the astronauts had varying opinions as to 
the usefulness and utility of ADA. Qualitatively, ADA was found to improve the damping response of the RMS but, overall, it was not a significant RMS operational performance improvement. Several of the astronauts felt that ADA had the most merit when maneuvering close to structure or performing precision tasks such as berthing. The added damping might allow for higher rate, precision maneuvers on-orbit. Active damping of the RMS was

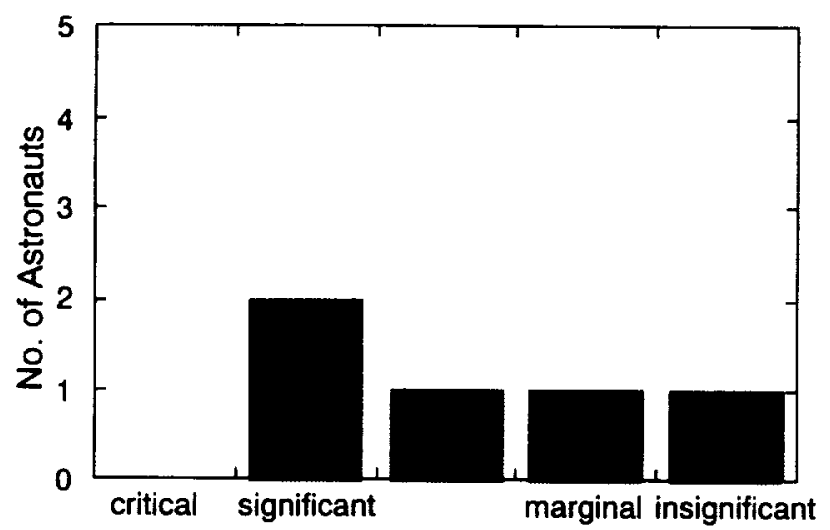

a) How would you rate the improvement in RMS damping?

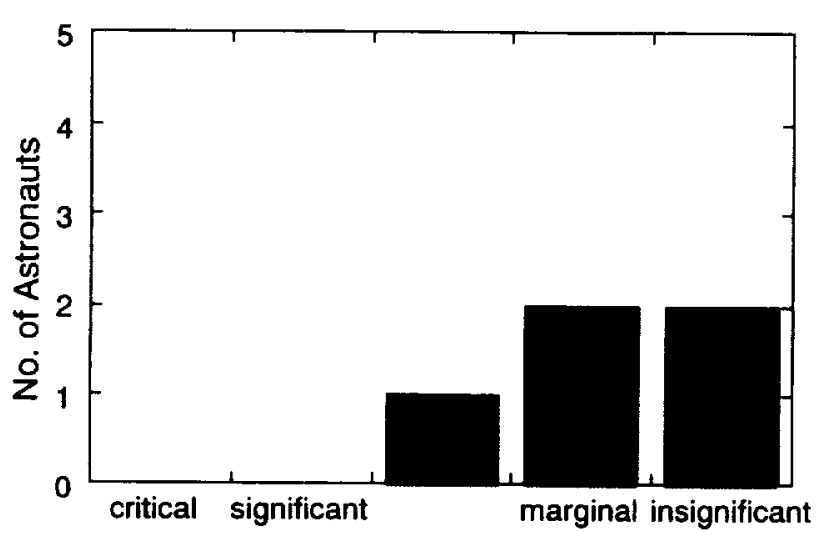

b) How would you rate the improvement in RMS operational performance?

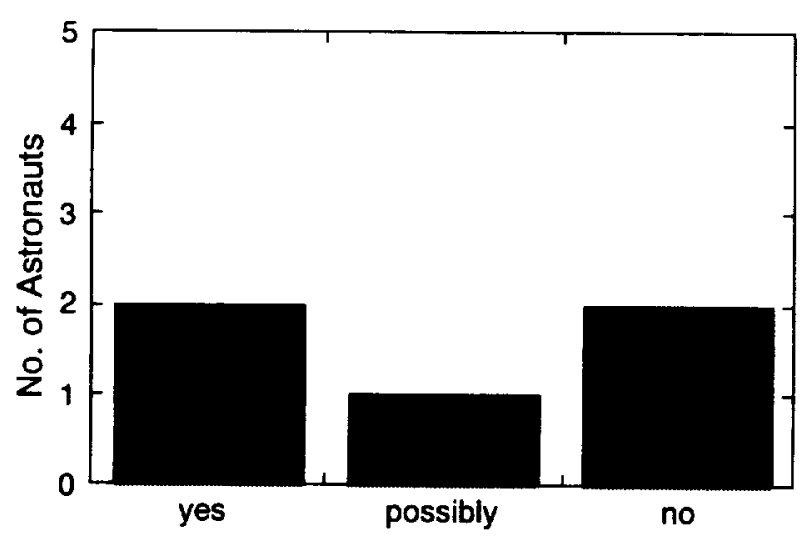

c) Would ADA be a benefit on orbit?

Figure 9. Astronaut Survey Results considered less critical when the payload is 'up and away' from the cargo bay.

\section{Quantitative results}

Quantitative results of the astronaut evaluation sessions in the SES were derived from recorded simulation data. Figure 10 depicts the typical activation of ADA for a $y$-axis translational hand controller maneuver. At the top is the ADA logic flag and the bottom is the $y$-axis translational hand controller maneuver command (in counts from \pm 88 ; where 88 corresponds to the full rate limit as given in Table 1). As shown, ADA is turned off when the maneuver command is initiated (at $\sim 649 \mathrm{sec}$ ) and then reactivated immediately following removal of the command (at -656.5 sec). Thus, ADA does not modify the commanded maneuver, (i.e., the way the arm "feels" to a trained operator), and is invoked at the end of the maneuver to improve the damping response of the RMS.
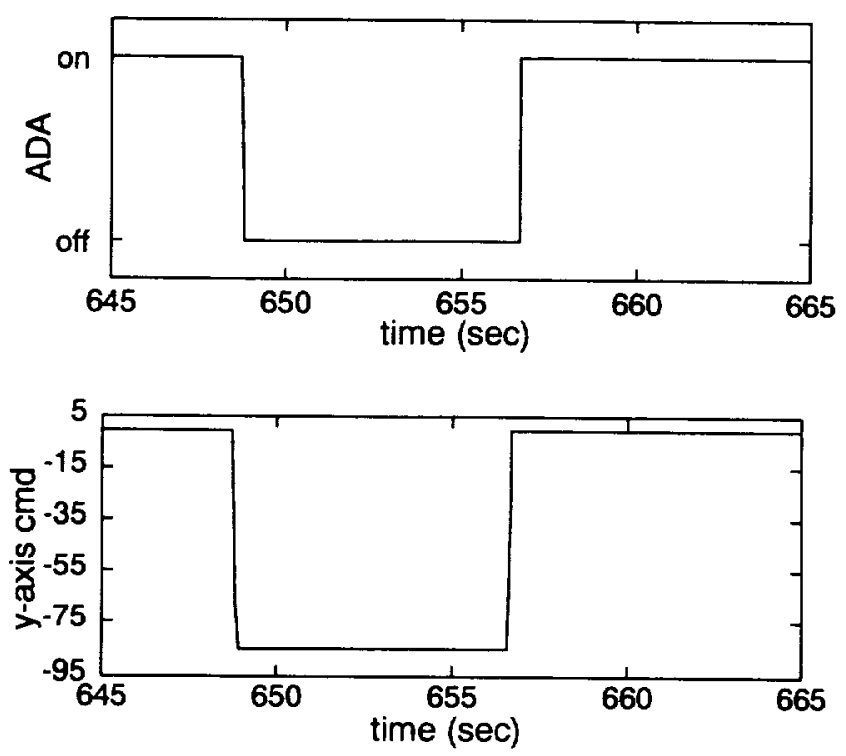

Figure 10: ADA activation

Figures 11 and 12 show typical results obtained for the MFR and HST payload, respectively. In both cases, the response to a $y$-axis translation command with and without ADA active are shown. Each set includes the magnitude (three-axis RSS) of the payload position, and the shoulder yaw joint servo torque. The set on the left-half of each figure are without $\mathrm{ADA}$ (cockpit switch turned off) and the set on the right are with ADA activated as described above. The time histories begin following completion of the operator commanded portion of the maneuver. For both the MFR and HST cases, it can be seen that ADA improves the damping of the position and torque response relative to the 'without-ADA' case. Damping of motion with the MFR payload was achieved much faster (in time) than the HST payload. This is due to the fact that the HST has a much larger inertia, a very low frequency fundamental mode, and significantly smaller joint rate limits. This heavy inertia makes it difficult to improve the first command overshoot response, however, ADA was effective at reducing 

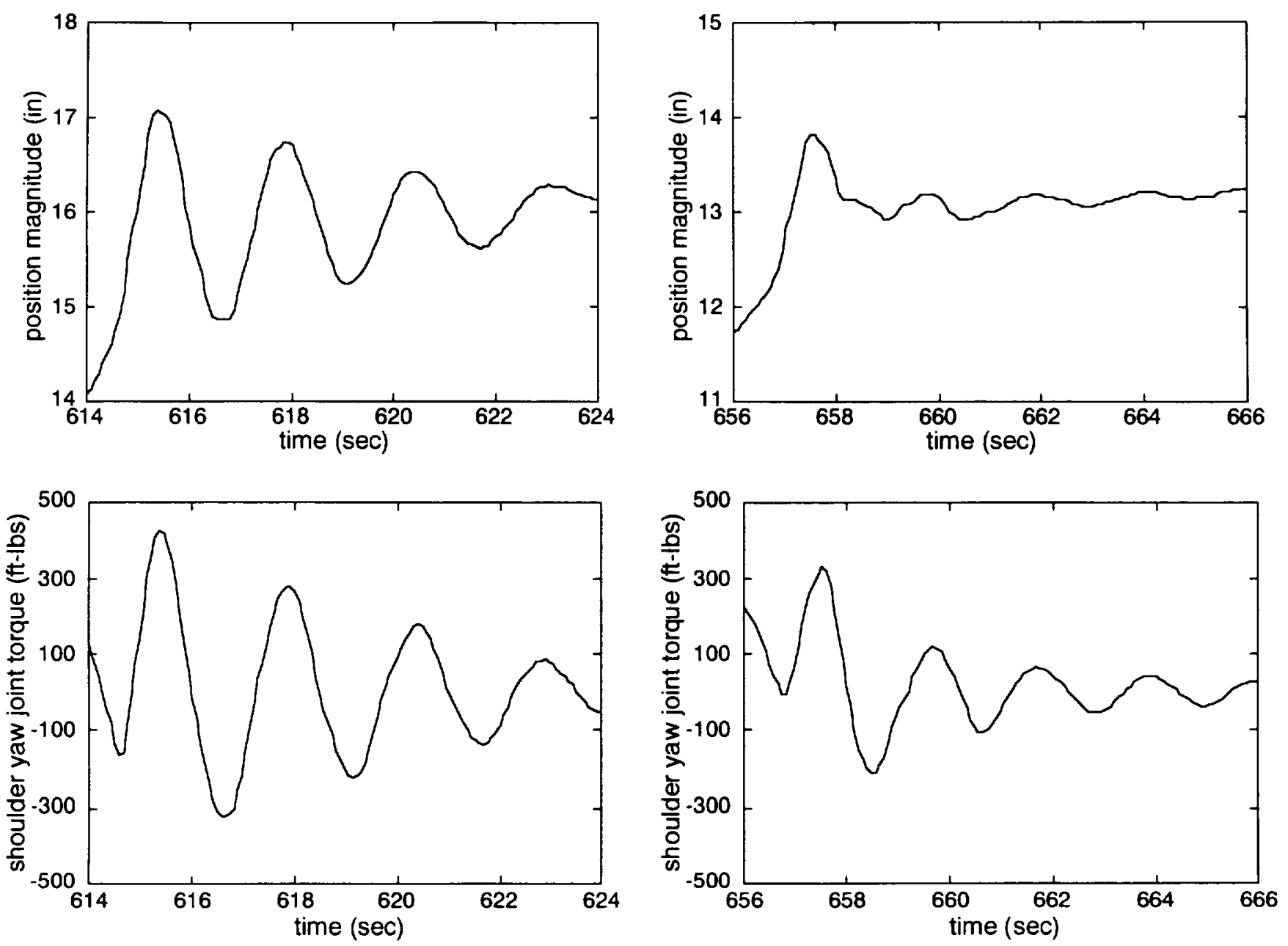

a) WITHOUT ADA

b) WITH ADA

Figure 11: MFR Results

subsequent oscillatory motion. Similar results were obtained for single joint, rotational, and combined rotational and translational operator commanded motions.

\section{Concluding Remarks}

CSI technology has been applied to the Shuttle RMS in order to improve its on-orbit performance. The objective was to actively damp the undesired oscillatory motions of the RMS following routine payload maneuvering and Shuttle attitude control thruster firings. Simulation of ADA was conducted in the real-time man-in-the-loop SES at the NASA Johnson Space Center with the objective of obtaining a qualitative definition of RMS operational performance improvement by astronaut operators and obtaining supporting quantitative performance data. Sensed vibratory motions were modeled using a simulated threeaxis accelerometer mounted at the end of the lower boom of the RMS. The sensed motions were feedback to an ADA control law, implemented in the Shuttle GPC, which generated commands to the joint servo mechanisms to reduce the unwanted oscillations. Based on the unanimous recommendation of astronaut operators who evaluated ADA applied to average (middle) weight payload, active damping of the RMS with attached heavy and very light payloads was successfully demonstrated. Five astronaut operators examined the performance of two ADA control laws following single-joint and six-joint coordinated translational and rotational maneuvers. Active Damping Augmentation disturbance rejection of Shuttle thruster firings was also evaluated. Noticeable improvements in the damping response of the RMS with both the heavy, HST, payload and very light, MFR, payload were observed. The potential of ADA to aid in maneuvering payloads close to structure or during precision tasks was deemed significant.

\section{Acknowledgments}

This study was the result of a very successful collaboration between several different organizations. The authors gratefully acknowledge individuals at The Charles Stark Draper Laboratory (Cambridge, MA), Lockheed Engineering \& Sciences Company (Houston, TX and 

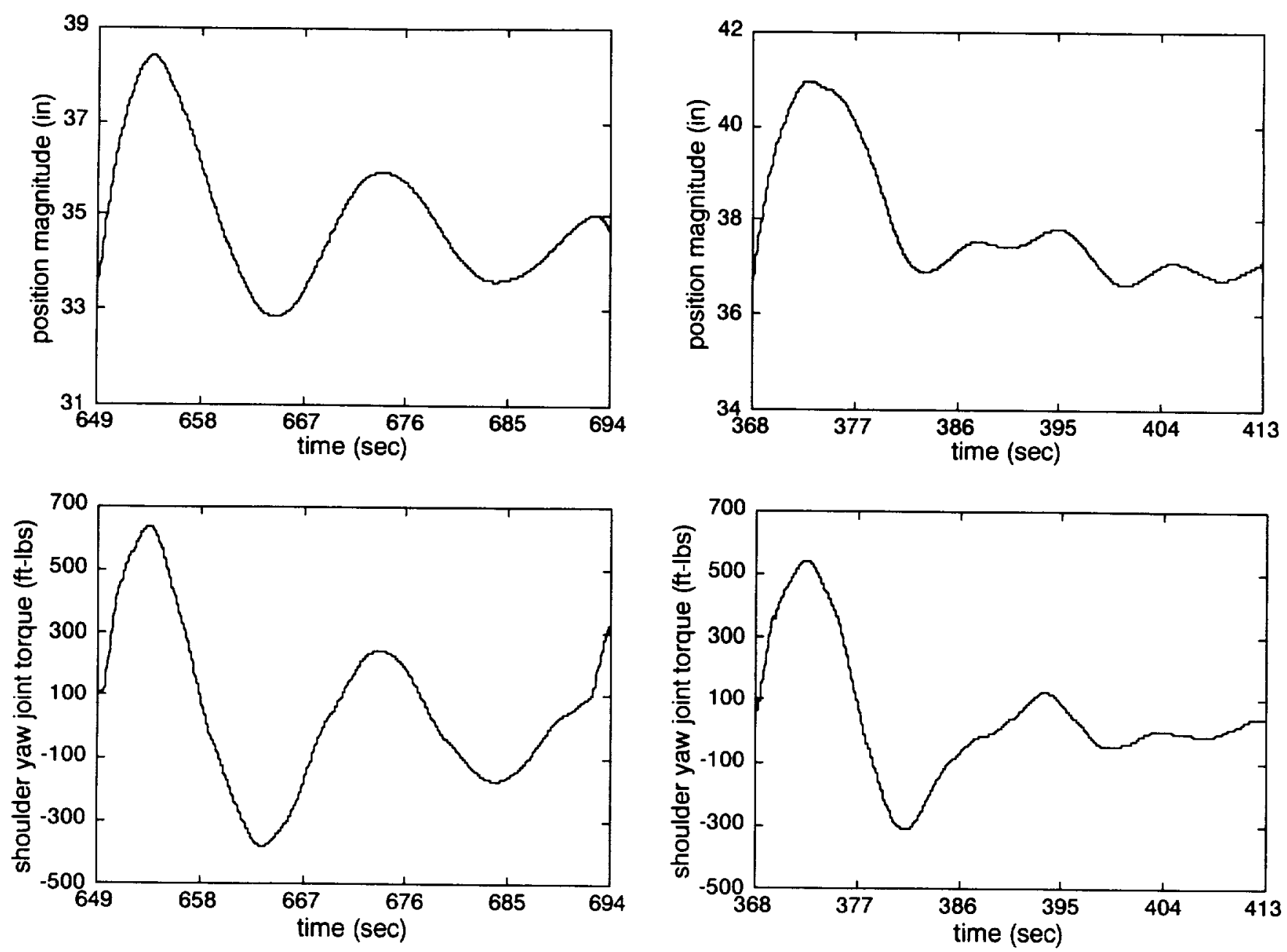

a) WITHOUT ADA

b) WITH ADA

Figure 12: HST Results

Hampton, VA), and the NASA Johnson Space Center (Houston, TX) for their contributions to this work.

\section{$\underline{\text { IX. References }}$}

1 Gilbert, Michael G.; Scott, Michael A.; and Sean P. Kenny: "Active Damping Augmentation to the Shuttle RMS”. NASA TM-102763, January 1991.

2 Scott, Michael A.; Gilbert, Michael G.; and Martha E. Demeo: “Active Vibration Damping of Space Shuttle Remote Manipulator System". Journal of Guidance, Control and Dynamics, Volume 16, Number 2, March-April 1993, pp. 275-280.

3 Demeo, M. E.; Gilbert, M. G.; Scott, M. A.; Lepanto, J. A.; Bains, E. M.; and Jensen, M. C.: Human-InThe-Loop Evaluation of RMS Active Damping Augmentation. AIAA 93-3875, Proceedings of the AIAA Guidance, Navigation, and Control Conference, Monterey, California, August 9-11, 1993. Accepted for publication, Journal of Guidance, Control and Dynamics.
4 "Space Shuttle System Payload Accommodations", NSTS 007700, Volume XIV, Appendix 8, Revision J, NASA Johnson Space Center, Houston, Texas, 1/27/88.

5 "Shuttle Flight Operations Manual: Vol. 16 Payload Deployment and Retrieval System", JSC-12770, Flight Operations Directorate, NASA Johnson Space Center, Houston, Texas, June 1, 1981.

6 Payload Deployment and Retrieval System Simulation Database, Version 1.0, RMS Operations; NASA JSC-25134, July 1, 1991.

7 Juang, Jer-Nan, Phan, Minh, Horta, Lucas G., and Richard W. Longman, "Identification of Observer/Kalman Filter Markov Parameters: Theory and Experiments", Journal of Guidance, Control and Dynamics, Volume 16, Number 2, March-April 1993, pp. 320-329.

8 Phan, Minh, Horta, Lucas G., Juang, Jer-Nan, and Richard W. Longman, "Linear System Identification Via an Asymptotically Stable Observer", AIAA 91-2735, Proceedings of the AIAA Guidance Navigation and Control Conference, New Orleans, Louisiana, August 1991. 
9 Juang, Jer-Nan, Horta, Lucas G., and Minh Phan, "System/Observer/Controller Identification Toolbox", NASA TM-107566, February 1992.

10 Horta, Lucas, G., Phan, Minh, Juang, Jer-Nan, Longman, Richard W., and Jeffrey L. Sulla, "Frequency Weighted System Identification and Linear Quadratic Controller Design", Journal of Guidance, Control and Dynamics, Volume 16, Number 2, March-April 1993, pp. 330-336.

11 Gupta, N. K., "Frequency-Shaped Cost Functionals: Extension of Linear-Quadratic Gaussian Design Methods", Journal of Guidance and Control, Volume 3, November-December 1980, pp. 529-535.

12 Doyle, John, "Analysis of Feedback Systems with Structured Uncertainties," IEEE Proceedings., Vol. 129, Pt. D, No. 6, Nov. 1982.

13 Fan, M. and Tits, A., "Characterization and Efficient Computation of the Structured Singular Value," IEEE Trans. on Auto. Control, Vol. AC-31, No. 8, 1986.

14 Doyle, J., Lenz, K., Packard, A.. "Design Examples Using $\boldsymbol{\mu}$-Synthesis: Space Shuttle Lateral Axis FCS During Reentry," Proceedings of 25th Conference on Decision and Control, December 1986.

15 Moore, B. C., "Principle Component Analysis in Linear Systems: Controllability, Observability and Model Reduction," IEEE Trans. On Auto. Control, Vol. AC-26, pp. 17-32, 1981

16 Gray, et. al., "Validation of the Draper RMS Simulation (DRS) Against Flight Data". CSDL-R-1755, Volume 1-2, The Charles Stark Draper Laboratory, Cambridge, Massachusetts, April, 1985.

17 "Systems Engincering Simulator Remote Manipulator Flight-to-Simulation Validation Report", LESC-30028, Lockheed Enginecring and Sciences Company, Houston, Texas, May 1992.

18 "Systems Engineering Simulator (SES) Requirements Document for the Remote Manipulator System (RMS) Damping Augmentation Study", LESC. 29879, Lockheed Engineering \& Sciences Company, Houston, Texas, February 1992. 


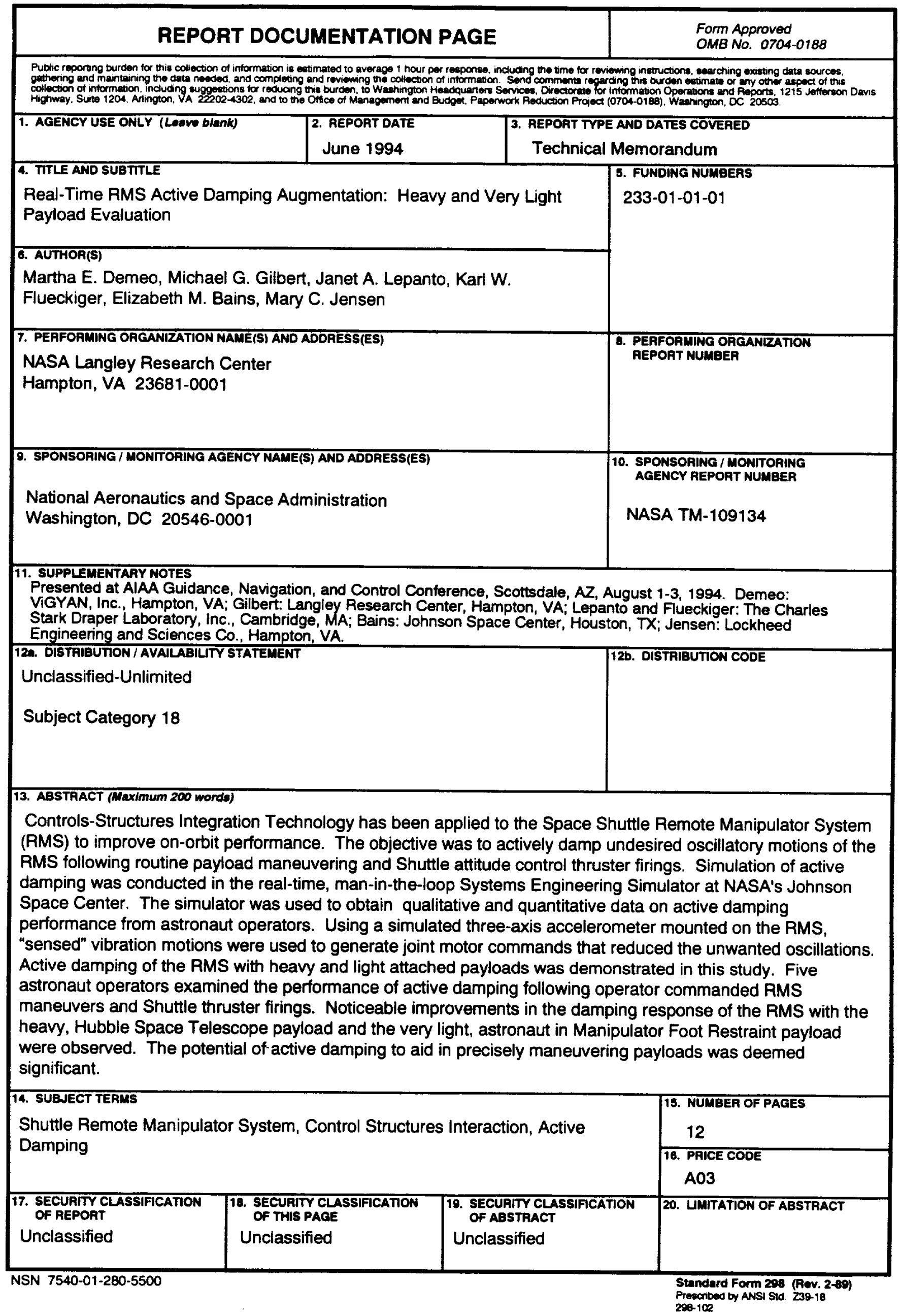

